\title{
Butterfly-like Heteronuclear 3d-4f Metal Clusters: Synthesis, Structures, Magnetic Properties, and Magnetocaloric Effect
}

\author{
Muhammad Nadeem Akhtar ${ }^{\dagger *}$, Murad A. AlDamen ${ }^{*}$, Magdalena Fitta ${ }^{\S}$, M. Shahid ${ }^{\perp}$, Alexander M. Kirillov ${ }^{\|, \#}$ \\ ${ }^{\dagger}$ Division of Inorganic Chemistry, Institute of Chemistry, Baghdad-ul-Jadeed Campus, The Islamia University of \\ Bahawalpur, Bahawalpur 63100, Pakistan. \\ tDepartment of Chemistry, School of Science, the University of Jordan, Amman 11942, Jordan. \\ §Institute of Nuclear Physics, Polish Academy of Sciences, 31-342 Kraków, Poland. \\ ${ }^{\perp}$ Functional Inorganic Materials Lab (FIML), Department of Chemistry, Aligarh Muslim University, Aligarh 202002, \\ India. \\ "Centro de Química Estrutural and Departamento de Engenharia Química, Instituto Superior Técnico, Universidade \\ de Lisboa, Av. Rovisco Pais, 1049-001, Lisbon, Portugal. \\ \#Research Institute of Chemistry, Peoples' Friendship University of Russia (RUDN University), 6 Miklukho-Maklaya \\ st., Moscow, 117198, Russian Federation. \\ Corresponding author: \\ nadeemchem@yahoo.com,m.nadeemakhtar@iub.edu.pk(M.N.Akhtar) \\ maldamen@ju.edu.jo (M. A. AlDamen)
}

\section{Table of Contents}

Materials and Methods ....................................................................................2

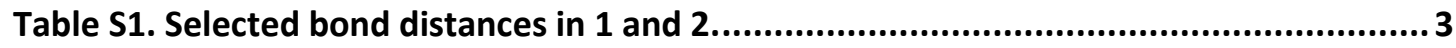

Figure S1. FTIR spectra of 1 and 2. ..................................................................4

Figure S2. Experimental and simulated powder X-ray diffraction (PXRD) patterns for 1 (above) and 2 (below)...............................................................................................5

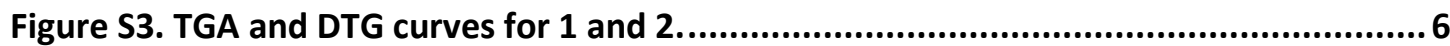

Figure S4. Ball-and-stick structure of 1. All hydrogen atom except those of $\mathrm{OH}^{-}$ligands are omitted for clarity. 7

Figure S5. The $\chi_{M}{ }^{-1}$ versus $T$ plots data for 1 and 2; inset shows the trend lines equations.....8

Figure S6. Magnetization curves for 1 and $2(T=2 \mathrm{~K})$. The dots are the best fitting results by the MagPack program and the line is the Brillouin fitting.....8

Figure S7. Energies $\left(\mathrm{cm}^{-1}\right)$ of the spin configurations of 1 (a) and 2 (b), obtained by MagPack package.9

Table S2. Estimated specific entropy change $-\Delta S_{m}$ upon application of magnetic field and temperature $T_{\text {max }}$ for magnetically isotropic Fe ${ }^{\prime \prime \prime}-\mathrm{Ln}^{\mathrm{III}}$ molecular clusters. 10 
Materials and Methods. All chemicals were obtained from available sources and were used as such without any further purification. All manipulations were executed under aerobic conditions. FTIR spectra were recorded with Bio-Rad FTS-spectrometers in the range of $4000-400 \mathrm{~cm}^{-1}$, and the samples were prepared as $\mathrm{KBr}$ pellets. Elemental analyses for Carbon, Hydrogen, and Nitrogen were carried out on Elementar Vario-ELCHNS elemental analyzer. PXRD data was collected on a Bruker D8 ADVANCE diffractometer with $\mathrm{Cu} \mathrm{K \alpha}$ radiation $(\lambda=1.54056 \AA)$, with a scan speed of $5^{\circ} \mathrm{min}^{-1}$ in the range of $2 \vartheta=$ 5-50 . TGA were carried out using a NETZSCH TG 209F1 Libra instrument under air atmosphere, by heating the samples from 29.2 to $900{ }^{\circ} \mathrm{C}$ with a heating rate of $1{ }^{\circ} \mathrm{C} \mathrm{min}{ }^{-1}$. Magnetic and magnetocaloric measurements were measured by using a superconducting quantum interference device (SQUID) magnetometer (Quantum Design MPMS-XL). The polycrystalline samples were enclosed in parafilm and immobilized with Vaseline to prevent the rotation of the sample. The DC magnetic susceptibility was measured under applied magnetic fields of 500 Oe. Isothermal magnetization curves, used for MCE evaluation, were measured at temperatures ranging from $2 \mathrm{~K}$ up to $50 \mathrm{~K}$ and in the field range of 0-7.0 T. The Pascal's constant was used for the diamagnetic corrections. 
Table S1. Selected bond distances in $\mathbf{1}$ and $\mathbf{2}$.

\begin{tabular}{|c|c|c|}
\hline Distances & 1: $\mathrm{Fe}_{6} \mathrm{Y}$ & 2: $\mathrm{Fe}_{6} \mathrm{Gd}$ \\
\hline Ln1-01 & $2.449(2)$ & $2.485(3)$ \\
\hline Ln1-02 & $2.443(2)$ & $2.479(3)$ \\
\hline Ln1-03 & $2.285(2)$ & $2.324(3)$ \\
\hline Ln1-05 & $2.261(2)$ & $2.302(3)$ \\
\hline Ln1-09 & $2.300(2)$ & $2.336(3)$ \\
\hline $\operatorname{Ln}_{1}-\mathrm{O} 11$ & $2.272(2)$ & $2.314(3)$ \\
\hline $\operatorname{Ln}_{1}-015$ & $2.423(2)$ & $2.465(3)$ \\
\hline $\operatorname{Ln}_{1}-\mathrm{O} 16$ & $2.451(2)$ & $2.490(3)$ \\
\hline Fe1-01 & $2.009(2)$ & $2.013(3)$ \\
\hline Fe1-O4 & $2.215(2)$ & $2.211(3)$ \\
\hline Fe1-05 & $1.980(2)$ & $1.982(3)$ \\
\hline Fe1-06 & $1.941(2)$ & $1.938(3)$ \\
\hline Fe1-07 & $1.941(2)$ & $1.947(3)$ \\
\hline $\mathrm{Fe} 2-\mathrm{O} 1$ & $1.995(2)$ & $1.992(3)$ \\
\hline $\mathrm{Fe} 2-\mathrm{O} 3$ & $1.987(2)$ & $1.986(3)$ \\
\hline $\mathrm{Fe} 2-\mathrm{O} 4$ & $2.243(2)$ & $2.251(3)$ \\
\hline $\mathrm{Fe} 2-08$ & $1.968(2)$ & $1.974(3)$ \\
\hline Fe3-O4 & $2.063(2)$ & $2.061(3)$ \\
\hline Fe3-07 & $2.014(2)$ & $2.011(3)$ \\
\hline Fe3-08 & $2.030(2)$ & $2.030(3)$ \\
\hline Fe4-02 & $2.018(2)$ & $2.017(3)$ \\
\hline Fe4-010 & $2.204(2)$ & $2.205(3)$ \\
\hline Fe4-011 & $1.989(2)$ & $1.986(3)$ \\
\hline $\mathrm{Fe} 4-012$ & $1.936(2)$ & $1.935(3)$ \\
\hline Fe4-013 & $1.942(2)$ & $1.944(3)$ \\
\hline $\mathrm{Fe} 5-\mathrm{O} 2$ & $2.000(2)$ & $0.997(3)$ \\
\hline Fe5-09 & $1.993(2)$ & $1.988(3)$ \\
\hline $\mathrm{Fe} 5-010$ & $2.228(2)$ & $2.228(3)$ \\
\hline $\mathrm{Fe} 5-\mathrm{O} 14$ & $1.966(2)$ & $1.964(3)$ \\
\hline Fe6-010 & $2.078(2)$ & $2.078(3)$ \\
\hline Fe6-013 & $2.019(2)$ & $2.018(3)$ \\
\hline Fe6-014 & $2.029(2)$ & $2.032(3)$ \\
\hline
\end{tabular}




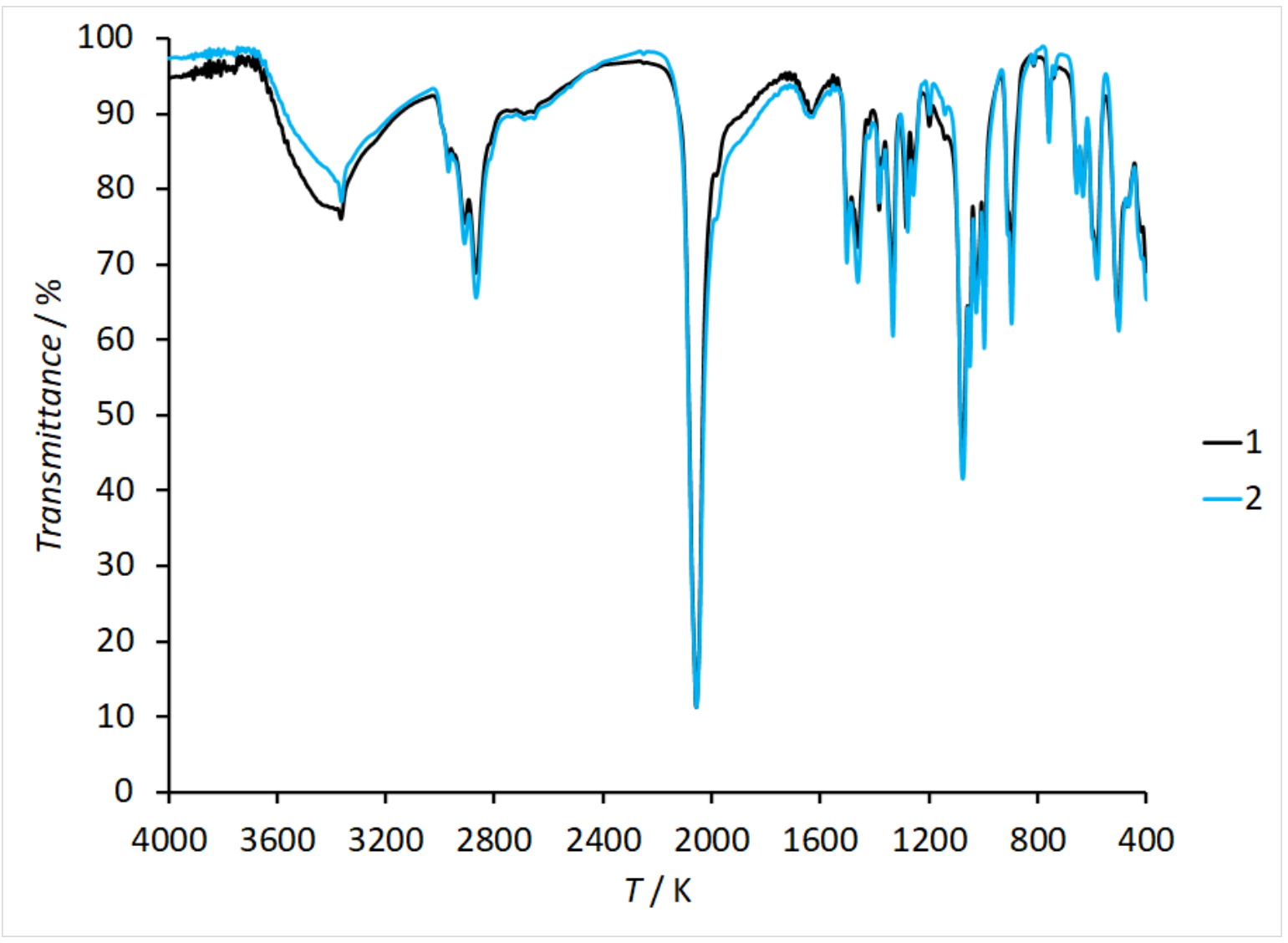

Figure S1. FTIR spectra of $\mathbf{1}$ and 2.

SH 

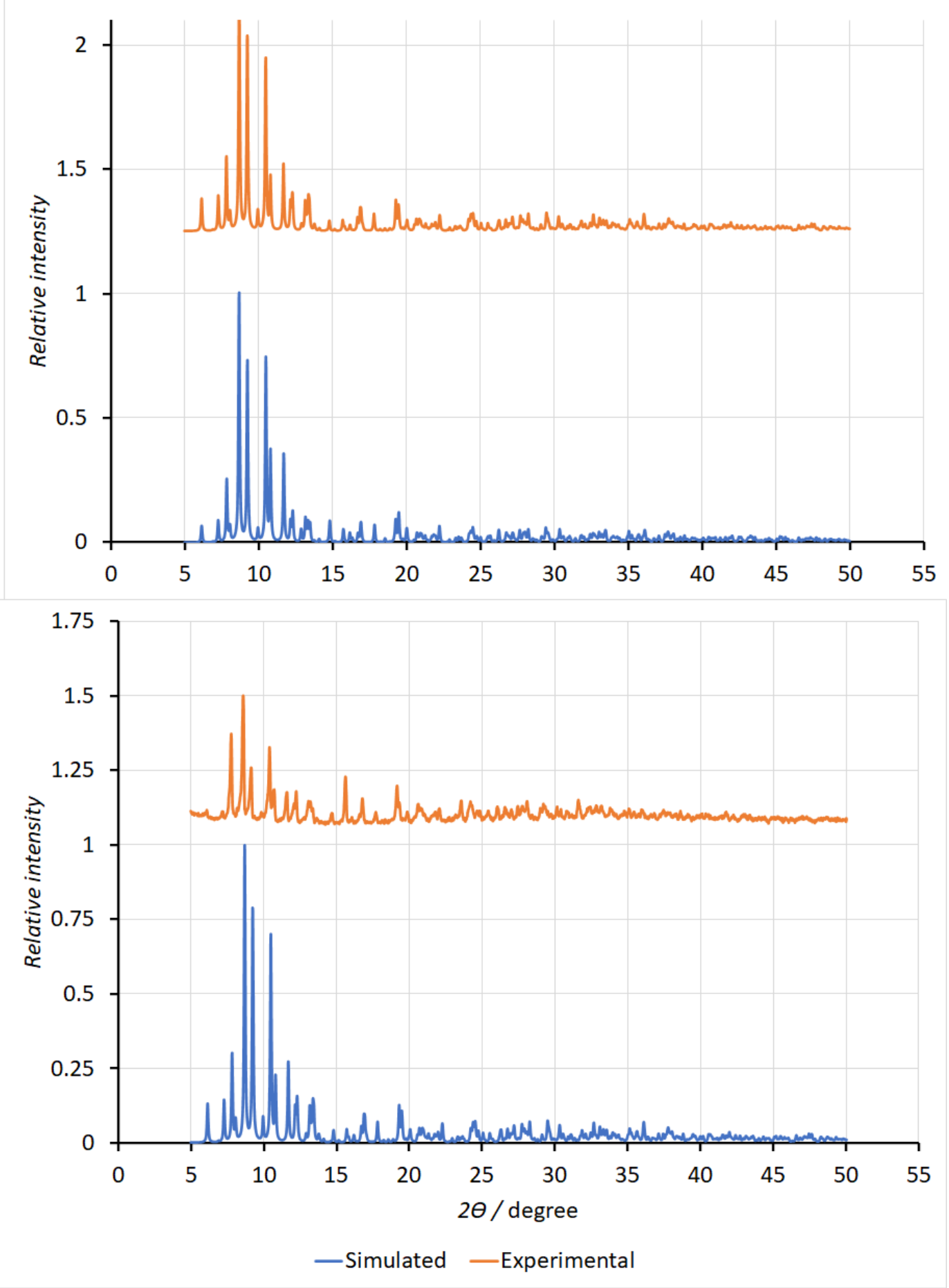

Figure S2. Experimental and simulated powder X-ray diffraction (PXRD) patterns for $\mathbf{1}$ (above) and 2 (below). 

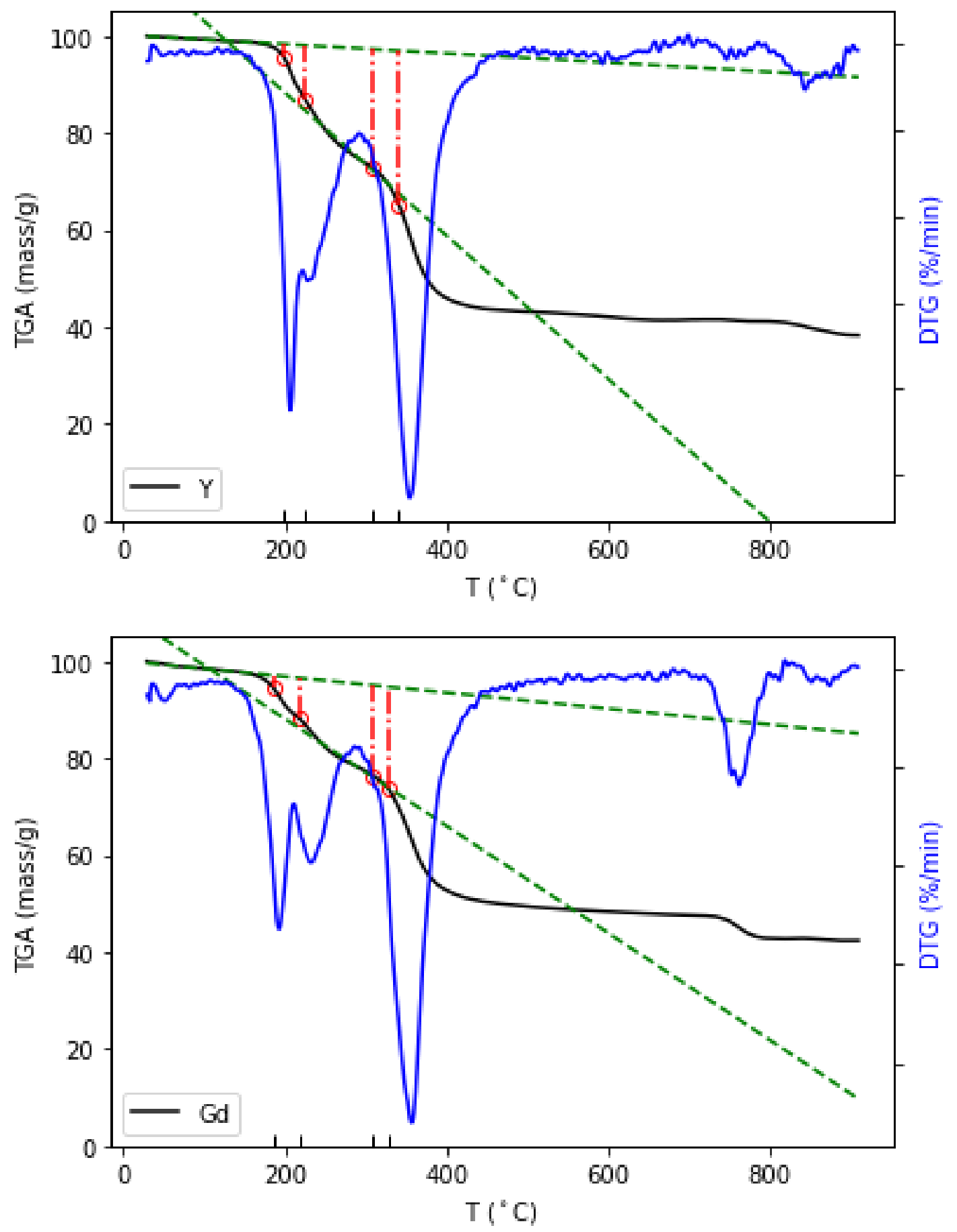

Figure S3. TGA and DTG curves for 1 and 2. 


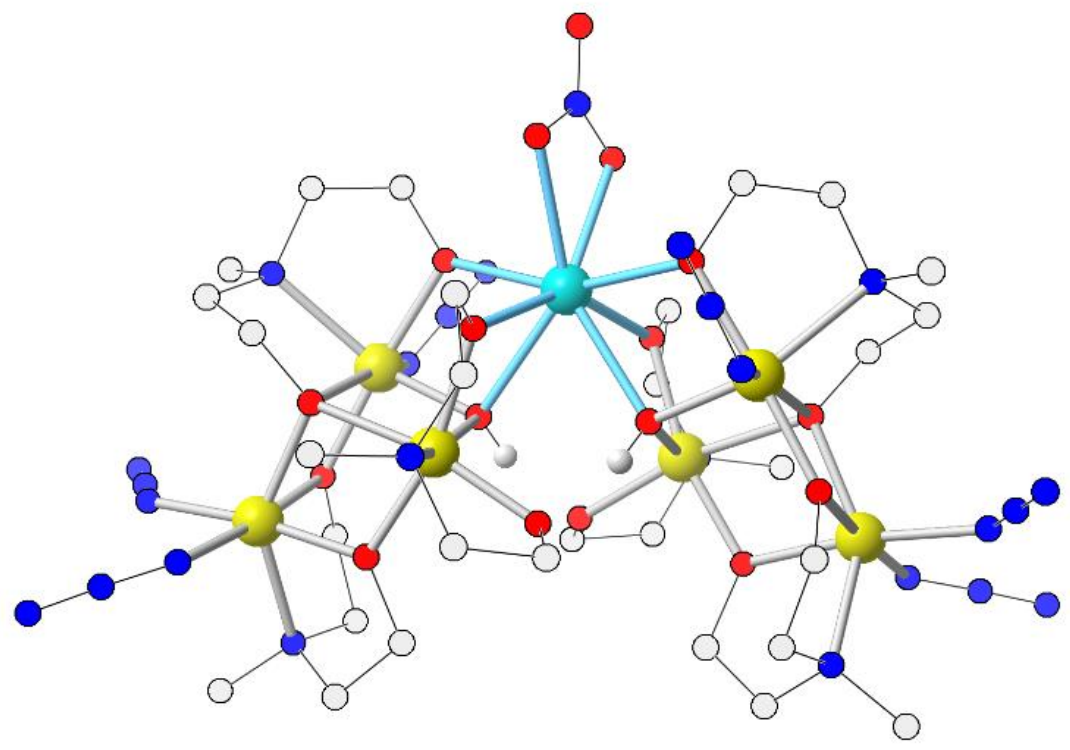

Figure S4. Ball-and-stick structure of 1. All hydrogen atom except those of $\mathrm{OH}^{-}$ligands are omitted for clarity. 


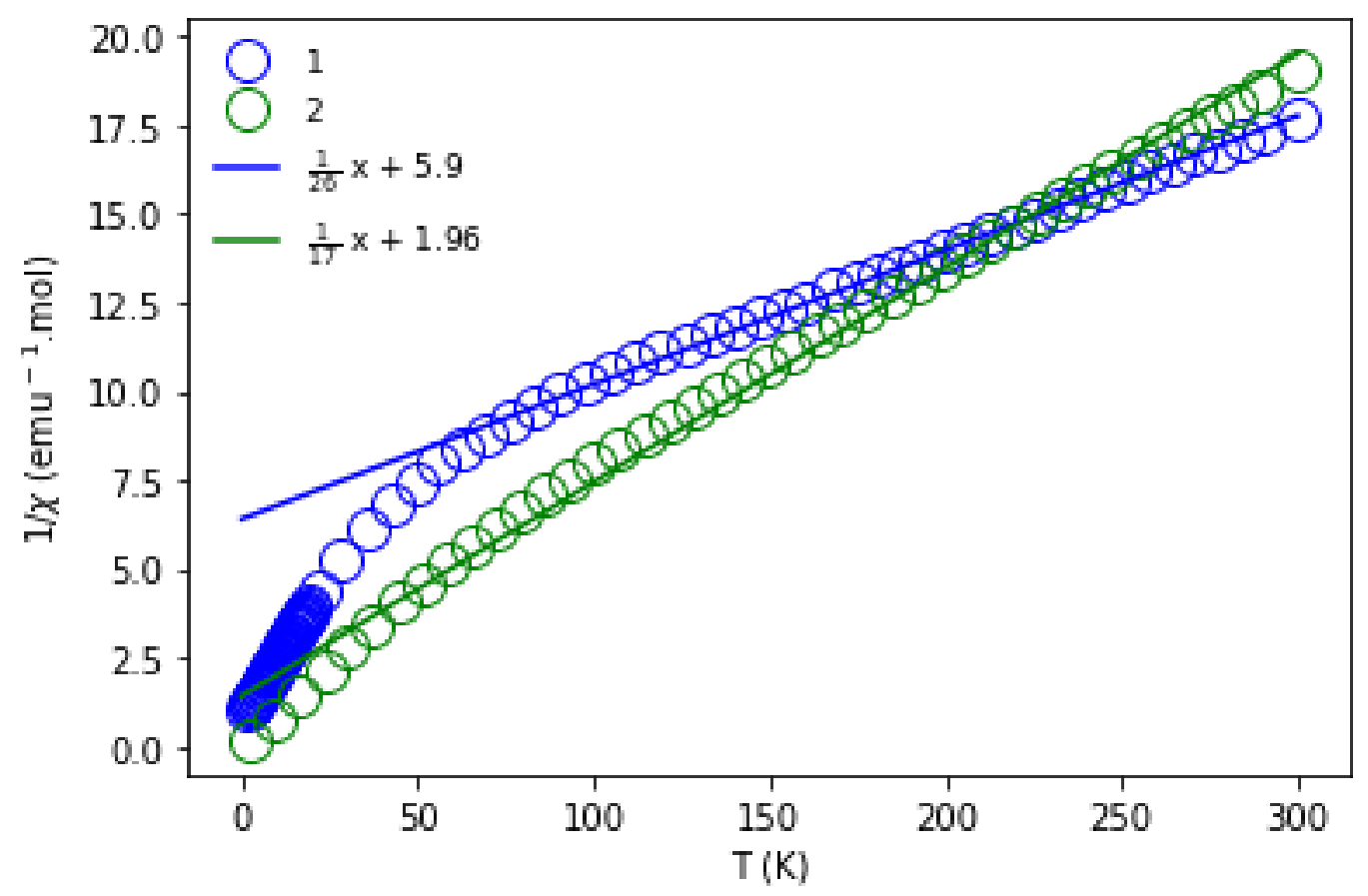

Figure S5. The $\chi_{M}{ }^{-1}$ versus $T$ plots data for $\mathbf{1}$ and $\mathbf{2}$; inset shows the trend lines equations.

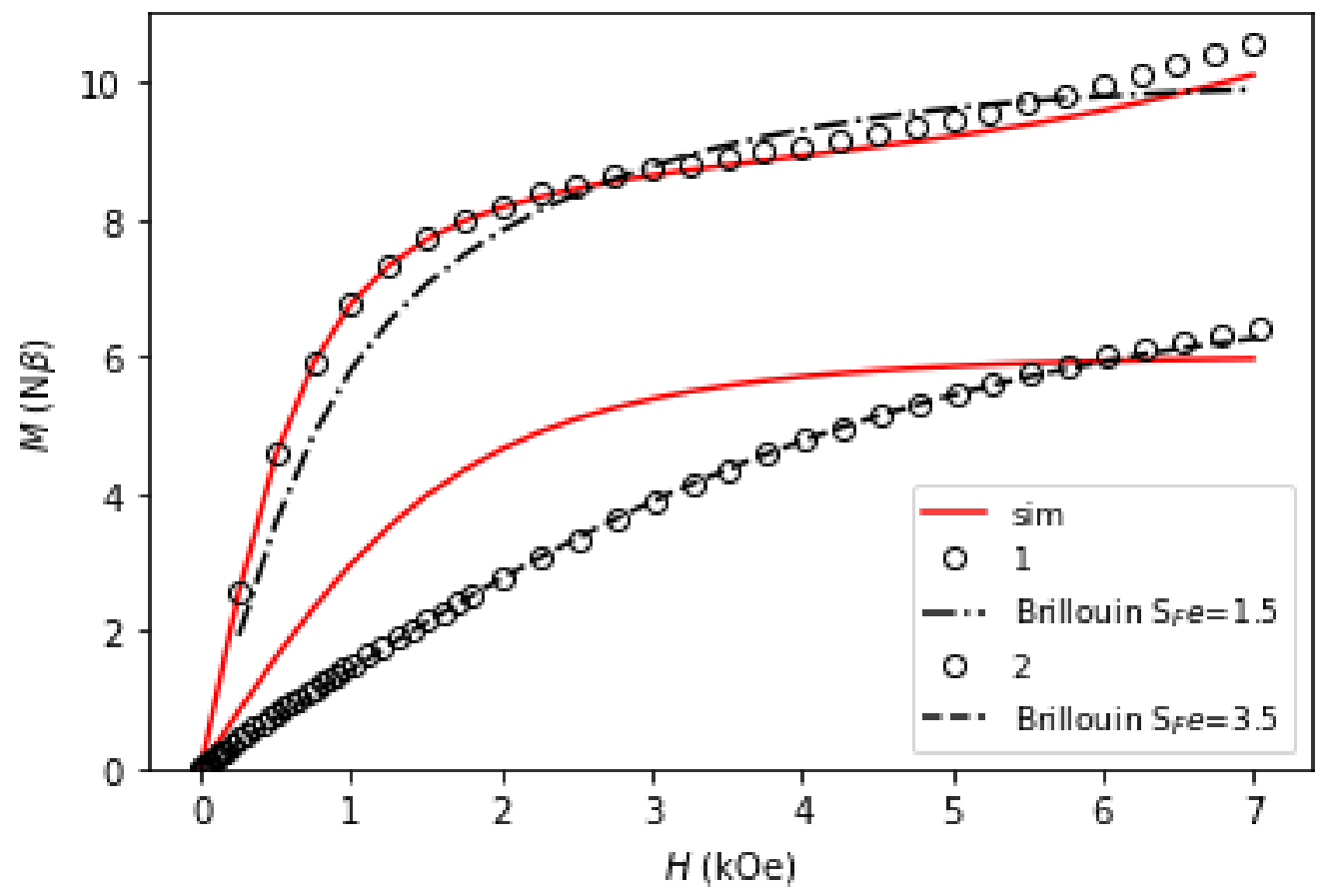

Figure S6. Magnetization curves for $\mathbf{1}$ and $\mathbf{2}(T=2 \mathrm{~K})$. The dots are the best fitting results by the MagPack program and the line is the Brillouin fitting. 

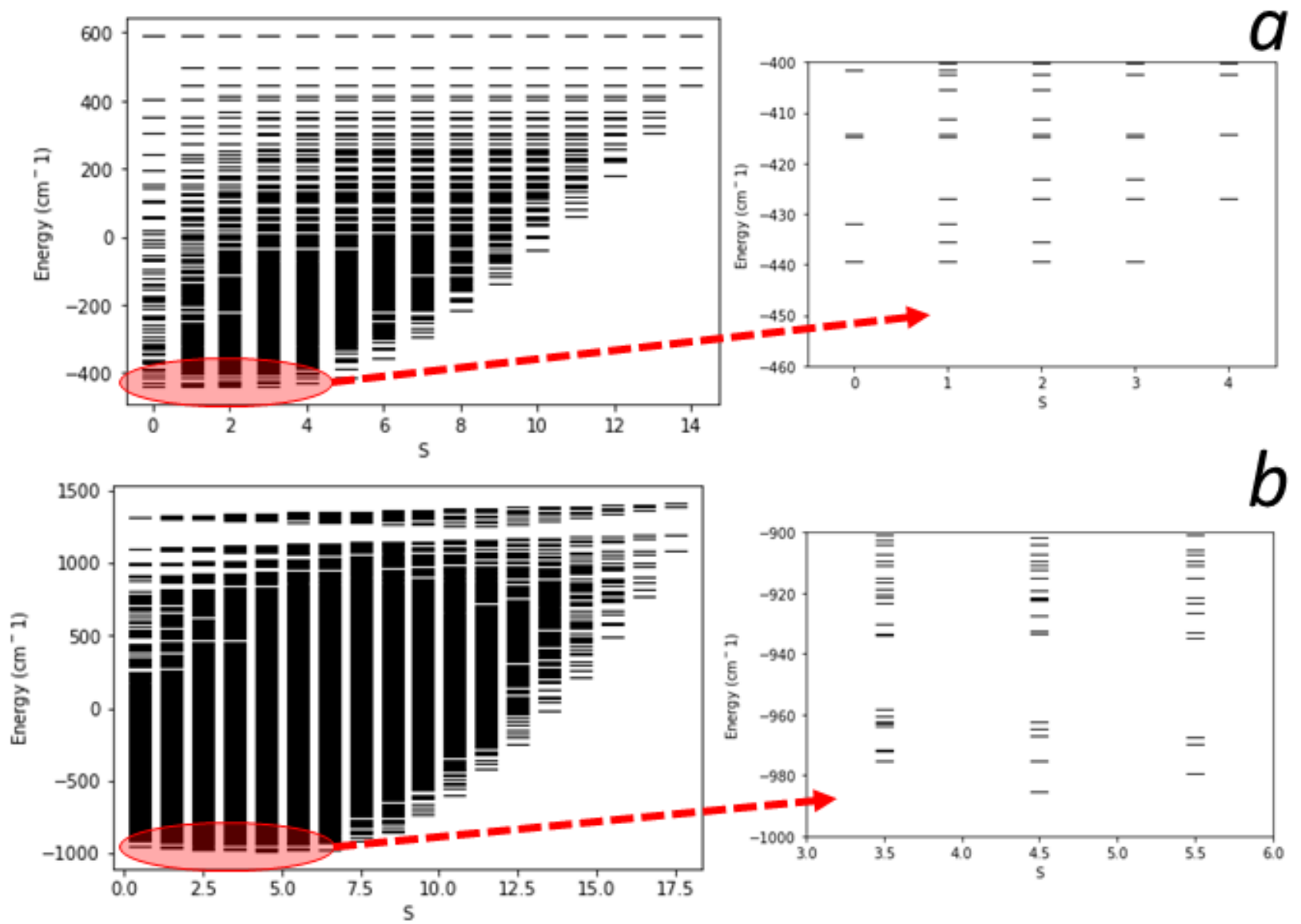

Figure S7. Energies $\left(\mathrm{cm}^{-1}\right)$ of the spin configurations of $\mathbf{1}(\mathrm{a})$ and $\mathbf{2}(\mathrm{b})$, obtained by MagPack package. 
Table S2. Estimated specific entropy change $-\Delta S_{m}$ upon application of magnetic field and temperature $T_{\text {max }}$ for magnetically isotropic Fe ${ }^{\text {III-Ln }}{ }^{\text {III }}$ molecular clusters.

\begin{tabular}{|c|c|c|c|c|}
\hline Core $^{a}$ & $-\Delta S_{m}\left(\mathrm{~J} \cdot \mathrm{kg}^{-1} \cdot \mathrm{K}^{-1}\right)$ & $T(\mathrm{~K})$ & $H(\mathrm{~T})$ & Ref. \\
\hline $\mathrm{Zn}_{8} \mathrm{Gd}^{\prime \prime \prime}{ }_{4}$ & 18.0 & 2.0 & 7 & S1 \\
\hline $\mathrm{Co}^{\prime \prime \prime \prime}{ }_{3} \mathrm{Gd}_{4}^{\prime \prime \prime}{ }_{4}$ & 24.9 & 2.0 & 7 & s2 \\
\hline $\mathrm{Fe}^{\mathrm{II \prime}}{ }_{6} \mathrm{Gd}^{\prime \prime \prime}{ }_{6} \mathrm{P}_{6}$ & $22.0-25.4^{b}$ & 3.0 & 7 & s3 \\
\hline $\mathrm{Fe}^{\mathrm{III}}{ }_{2} \mathrm{Gd}^{\prime \prime \prime}{ }_{3}$ & 31.7 & 3.0 & 7 & S4 \\
\hline $\mathrm{Fe}^{\prime \prime \prime}{ }_{2} \mathrm{Gd}^{\prime \prime \prime}{ }_{3}$ & 33.1 & 4.2 & 7 & s5 \\
\hline $\mathrm{Fe}_{6}{ }_{6} \mathrm{Gd}^{\prime \prime \prime}{ }_{4}$ & 13.7 & 4.5 & 9 & s6 \\
\hline $\mathrm{Fe}^{\prime \prime \prime}{ }_{3} \mathrm{Gd}_{2}{ }^{\prime \prime \prime}{ }_{2}$ & 21.1 & 6.0 & 7 & S7 \\
\hline $\mathrm{Ni}_{8}{ }_{8} \mathrm{Gd}^{\prime \prime \prime}{ }_{4}$ & 22.0 & 3.6 & 7 & 58 \\
\hline $\mathrm{Mn}_{4}{ }_{4} \mathrm{Gd}^{\prime \prime \prime \prime}{ }_{4}$ & 19.0 & 4.0 & 7 & s9 \\
\hline $\mathrm{Cr}^{\prime \prime \prime \prime}{ }_{4} \mathrm{Gd}^{\prime \prime \prime \prime}$ & 18.3 & 3.5 & 8 & s10 \\
\hline $\mathrm{Gd}^{\prime \prime \prime} \mathrm{Fe}^{\prime \prime \prime}{ }_{4}{ }^{\mathrm{c}}$ & $6.1-7.3$ & $3-4$ & 7 & s11 \\
\hline 2 & 10.6 & 2.0 & 7 & This work \\
\hline
\end{tabular}

${ }^{a}$ The clusters are named according to their metal cores. ${ }^{b}$ Phosphonate substituent $=\mathrm{Me} v s . \mathrm{Ph}$, hexyl. ${ }^{\mathrm{c}}$ Two metallocrown clusters $\mathrm{PyH}\left[\mathrm{Fe}_{4} \mathrm{Gd}(\mathrm{shi})_{4}\left(\mathrm{PhCO}_{2}\right)_{4}(\mathrm{Py})_{4}\right] \cdot(\mathrm{MeOH})$ and $\left[\mathrm{Fe}_{4} \mathrm{Gd}(\mathrm{shi})_{4}\left(\mathrm{H}_{2} \mathrm{shi}\right)_{3}(\mathrm{Py})_{3}\left(\mathrm{H}_{2} \mathrm{O}\right)\right] \cdot(\mathrm{Py})$.

S1. Hooper, T. N.; Schnack, J.; Piligkos, S.; Evangelisti, M.; Brechin, E. K., The Importance of Being Exchanged: $\left[\mathrm{Gd}^{\prime \prime \prime}{ }_{4} \mathrm{M}_{8}{ }_{8}(\mathrm{OH})_{8}(\mathrm{~L})_{8}\left(\mathrm{O}_{2} \mathrm{CR}\right)_{8}\right]^{4+}$ Clusters for Magnetic Refrigeration. Angew. Chem., Int. Ed. 2012, 51, 4633-4636.

S2. Tian, C. B.; Yuan, D. Q.; Han, Y. H.; Li, Z. H.; Lin, P.; Du, S. W., Synthesis, structures, and magnetic properties of a series of new heterometallic hexanuclear Co2Ln4 ( $\mathrm{Ln}=\mathrm{Eu}, \mathrm{Gd}$, Tb and Dy) clusters. Inorg. Chem. Front. 2014, 1, 695-704.

S3. Pineda, E. M.; Tuna, F.; Zheng, Y. Z.; Teat, S. J.; Winpenny, R. E.; Schnack, J.; McInnes, E. J., Iron lanthanide phosphonate clusters: \{Fe6Ln6P6\} Wells-Dawson-like structures with D3d symmetry. Inorg. Chem. 2014, 53, 3032-3038.

S4. Li, H.; Shi, W.; Niu, Z.; Zhou, J.-M.; Xiong, G.; Li, L.-L.; Cheng, P., Remarkable LnIII3FellI2 clusters with magnetocaloric effect and slow magnetic relaxation. Dalton Trans. 2015, 44, 468-471.

S5. Pedersen, K. S.; Lorusso, G.; Morales, J. J.; Weyhermüller, T.; Piligkos, S.; Singh, S. K.; Larsen, D.; Schau-Magnussen, M.; Rajaraman, G.; Evangelisti, M.; Bendix, J., Fluoride-Bridged \{GdIII3MIII2\} (M = Cr, Fe, Ga) Molecular Magnetic Refrigerants. Angew. Chem., Int. Ed. 2014, 53, 2394-2397.

S6. Akhtar, M. N.; AlDamen, M. A.; Khan, J.; Shahid, M.; Kirillov, A. M., Heterometallic (3d-4f) Coordination Clusters with Unique Topology: Self-Assembly Synthesis, Structural Features, and Magnetic Properties. Cryst. Growth Des. 2020, 20, 6545-6554.

S7. Schmidt, S. F. M.; Koo, C.; Mereacre, V.; Park, J.; Heermann, D. W.; Kataev, V.; Anson, C. E.; Prodius, D.; Novitchi, G.; Klingeler, R.; Powell, A. K., A Three-Pronged Attack To Investigate the Electronic Structure of a Family of Ferromagnetic Fe4Ln2 Cyclic Coordination Clusters: A Combined Magnetic Susceptibility, High-Field/High-Frequency Electron Paramagnetic Resonance, and ${ }^{57}$ Fe Mössbauer Study. Inorg. Chem. 2017, 56, 4796-4806.

S8. Chakraborty, A.; Goura, J.; Kalita, P.; Swain, A.; Rajaraman, G.; Chandrasekhar, V., Heterometallic 3d-4f single molecule magnets containing diamagnetic metal ions. Dalton Trans. 2018, 47, 8841-8864.

S9. Dolomanov, O. V.; Bourhis, L. J.; Gildea, R. J.; Howard, J. A. K.; Puschmann, H., OLEX2: A complete structure solution, refinement and analysis program. Journal of Applied Crystallography 2009, 42, 339-341.

S10. Blacque, O.; Amjad, A.; Caneschi, A.; Sorace, L.; Car, P.-E., Synthesis, structure, magnetic and magnetocaloric properties of a series of \{CrIIILLnIII\} complexes New J. Chem., 2016, 40, 3571-3577.

S11. Salerno, E. V.; Kampf, J. W.; Pecoraro, V. L.; Mallah, T., Magnetic properties of two GdIIIFelll4 metallacrowns and strategies for optimizing the magnetocaloric effect of this topology. Inorg. Chem. Front. 2021. doi.org/10.1039/D1QI00207D 